\title{
Norois
}

Environnement, aménagement, société

\section{Exploration de récits paysagers sur les littoraux de Belle-île-en-Mer (France) et de la péninsule de Dingle (Irlande)}

Exploring landscape narratives on the coasts of Belle-Île-en-Mer (France) and Dingle Peninsula (Ireland)

\section{Helen Maulion}

\section{OpenEdition}

Journals

Édition électronique

URL : http://journals.openedition.org/norois/3008

DOI : $10.4000 /$ norois.3008

ISBN : 978-2-7535-1561-1

ISSN : 1760-8546

Éditeur

Presses universitaires de Rennes

\section{Édition imprimée}

Date de publication : 15 décembre 2009

Pagination : 41-57

ISBN : 978-2-7535-1050-0

ISSN : 0029-182X

Référence électronique

Helen Maulion, «Exploration de récits paysagers sur les littoraux de Belle-île-en-Mer (France) et de la péninsule de Dingle (Irlande) », Norois [En ligne], 213 | 2009/4, mis en ligne le 15 décembre 2011, consulté le 19 avril 2019. URL : http://journals.openedition.org/norois/3008 ; DOI : 10.4000/ norois.3008 


\title{
EXPLORATION DE RÉCITS PAYSAgERS SUR LES LiTTORAUX \\ de Belle-Île-en-Mer (France) \\ et de la péninsule de Dingle (Irlande)
}

\author{
Helen Maulion \\ Géolittomer - LETG UMR 6554 CNRS \\ (Université de Nantes), \\ Château du Tertre, BP 81227 - 44312 NANTES Cedex \\ Geography Department, UCC (Cork, Irlande) \\ helen.maulion@gmail.com
}

\section{RÉSUMÉ}

Cet article sintéresse à la complexité des connexions sensibles aux paysages littoraux de Belle-île-en-mer (France) et de la péninsule de Dingle (Irlande). Si elles correspondent parfois à une émotion esthétique, elles correspondent aussi à la recherche et à l'expérience d'un bien-être. La méthode d'enquête qualitative mise en place se décline par le recueil de récits paysagers, introduits par la réalisation d'une cartographie appliquée aux paysages, qui articule sentiments, mémoire et expériences corporelles. Le paysage n'est pas seulement abordé comme une représentation mais comme une relation ou un rapport au monde performatif, émotionnel et métaphorique. Ce concept est déployé à travers le corps sensible et l'engagement de l'habitant au monde.

MOTS CLÉ : paysage - récit - corporalité - géographie relationnelle - littoral - Irlande - France

\section{ABSTRACT \\ Exploring landscape narratives on the coasts of Belle-île en mer (France) and Dingle Peninsula (Ireland)}

This paper explores the complexity of the connections people make with coastal landscapes of Belle-île en mer (France) and the Dingle Peninsula (Ireland). Sometimes based on esthetical emotions, these connections are also present in the search for the experience of well-being. Using landscape mapping as a qualitative approach, I collected narratives that interlaced feelings, memories, everyday practices and embodied experiences. From the body to the engagement of dwelling in the world, landscape is therefore not approached as an idea but as a performative, emotional and metaphorical relation to the world

KEY WORDS : landscape - narrative - embodiment - relational geography - coast - Ireland-France 
À partir de l'analyse de récits paysagers, cet article présente les premiers résultats d'une recherche doctorale et fait dialoguer deux approches pour explorer les relations sensibles aux littoraux. La première articule une géographie de la relation qui interroge, au-delà des représentations, les expériences paysagères et leur rôle dans une relation paysagère performative, c'est-àdire qui se construit aussi à travers le récit qui en est fait. Le paysage est défini dans cet article comme une relation ou une médiation (Berque, 2000a), affective, sensible et en construction entre l'être et le monde. La dimension sensible est contenue dans le mouvement du corps en contact avec le milieu. C'est le corps expérientiel qui permet de créer et de nourrir la relation paysagère individuelle mais aussi sociale. La deuxième approche développée dans cette communication est phénoménologique. Dans la tradition géographique, ce positionnement humaniste et ontologique n'est pas nouveau (Berque, 2000a ; Buttimer, 1976; Dardel, 1952 ; Hoyaux, 2000 ; Tuan, 1974). Elle permet de réfléchir à la possibilité de dépasser le clivage moderne entre le sujet et l'objet sur lequel est fondé le concept occidental moderne de paysage (Berque, 2008) qui entraîne une série de tensions décrites par John Wylie (2007) reposant sur l'opposition entre culture et nature, proximité et distance, l'observer et l'habiter. Ainsi le paysage, raconté et construit dans les récits paysagers, est compris au sens large, comme une relation au monde, sensible, affective et intentionnelle, un « déploiement » comme dirait Eric Dardel (1990, p. 42) qui compose, avec les manières d'habiter et les processus territoriaux, «le territoire de l'être au monde » (Hoyaux, 2000, p. 118).

Mésologique (Bousquet, 1990), le littoral est un ensemble spatial défini de diverses manières : territoriale, législative, environnementale. On peut y ajouter l'horizon. Ici l'horizon n'est pas tant la ligne de vue que la profondeur qui nous en distance. Cette profondeur nous permet de considérer le mouvement du monde comme appréhendable à travers une tactilité qui peut être aussi visuelle (Merleau-Ponty, 1964). Le corps porte la perception et le mouvement qui traversent et animent topographies et lieux et contribuent à la relation paysagère. Le littoral est un espace liminaire (Corbin, 2005) ; c'est-à-dire un espace de contact et de seuil, où l'horizon exacerbe la relation paysagère. Le paysage littoral a été représenté à différentes époques sous la forme picturale ou littéraire. Ces images ont contribué à l'appréhension du paysage littoral, les images influençant les expériences esthétiques de la mer comme Alain Corbin (1988) l'a montré dans son étude et l'évolution des représentations littorales a accompagné l'évolution des pratiques littorales, notamment touristiques. Le littoral d'aujourd'hui n'est plus celui de l'époque romantique, ni celui des premiers congés payés et la relation aux paysages littoraux a changé. Réfléchir sur le paysage littoral, c’est réfléchir à deux « rendez-vous » contemporains à la fois sociétal et environnemental, celui avec le paysage et celui avec les milieux littoraux. Ces «rendez-vous » sont animés par les réflexions sur un développement durable ou soutenable et l'appel à une re-cosmisation de notre rapport au monde, poétique et éthique (Berque, 1996, 2000a, 2000b, 2008; White, 1994).

L'échelle individuelle du paysage est définie par Y. Luginbühl (2001) dans son analyse de la demande sociale de paysage. L'individu «investit les paysages connus de significations relatives à sa propre trajectoire, aux événements qu'il a vécus et par rapport à l'ensemble des paysages qu'il connaît ou qu'il a vus ou vécus » (Luginbühl, 2001, p. 2). Se saisissant de l'échelle individuelle, cet article explore le moment toujours renouvelé de la rencontre avec le milieu littoral et la manière dont cette rencontre est racontée en se demandant si le récit paysager participe à la compréhension de la relation au monde, à la fois expérience, performance, forme d'expérience réflexive avec pour spectateur soi-même, et représentations.

Dans cet article, j'explore les dimensions intime et émotionnelle, expérientielle et corporelle de la relation paysagère à travers des récits performatifs d'habitants et de touristes, récits qui font paysage. Il s'agit de présenter plusieurs pistes de réflexion en cours suggérées par l'analyse d'entretiens pour participer au débat sur le concept de paysage, à une échelle individuelle, sujette à caution et parfois difficile à inclure dans l'agenda social et politique. La première partie de cette communication présente les méthodes d'enquête et d'analyse mises en œuvre pour cette recherche doctorale; 
la deuxième partie explore comment le corps anime la relation paysagère, et enfin, la troisième partie réfléchit sur l'engagement présent dans le rapport au monde et les récits paysagers.

\section{Cartes paysagères : méthode expérimentale pour explorer les connections sensibles aux paysages}

Des lignes de force (Thrift, 2004) sont formées par les émotions et les sentiments, la mémoire et la connaissance et elles sont animées par le corps en mouvement. La relation paysagère ne peut s'étudier seulement par l'interprétation symbolique des représentations paysagères. Le paysage n'est pas appréhendé comme une 'manière de voir' (Cosgrove, 1998), mais comme une « manière de faire » ou un mouvement sensible et intime entre l'individu et son milieu en construction permanente. Afin d'analyser cette dynamique paysagère et ces lignes de forces, une méthode d'enquête qualitative et participative a été mise en place. L'enquête s'est déroulée dans deux zones ateliers (Figure 1) : Belle-île-en-Mer (5000 hab. environ) et l'ouest de la péninsule de Dingle (6000 hab. environ). Ce sont des espaces littoraux où se confrontent deux ensembles fortement marqués : la mer et les terres intérieures, caractérisées par un plateau et des vallons à Belle-île et par plusieurs sommets à Dingle (Mont Brandon culmine à $952 \mathrm{~m}$ et Mont Eagle à $512 \mathrm{~m}$ ) qui s'opposent et se complètent d'un point de vue paysager. Les deux ensembles sont extrêmement touristiques avec comme attraction la qualité des paysages et la relative protection de l'aspect 'naturel' et 'sauvage'.

L'enquête, réalisée entre juillet 2007 et mars 2008, comportait deux phases. Une première rencontre permettait de remettre une carte et un dossier aux participants et de présenter le projet de recherche. L'entretien se faisait pendant la deuxième rencontre, une fois la carte réalisée ${ }^{l}$. Plusieurs personnes souhaitant participer à l'enquête m'ont contacté suite à des affiches et des articles de journaux qui ont permis de diffuser l'information sur ce travail de recherche. Le bouchea-oreille a ensuite permis de rencontrer d'autres personnes. Cette enquête se voulait qualitative et participative, c'est-à-dire que les personnes rencontrées n'étaient pas des objets de recherche mais bien des sujets aux trajectoires personnelles, qui allaient raconter leurs liens aux paysages de l'île ou de la péninsule.

\section{De LA CARTE À L'ENTRETIEN}

En développant une méthode s'appuyant sur la carte, l'objectif était d'encourager les participants à réfléchir sur leurs connexions sensibles aux lieux et de les traduire en récit. 28 personnes à Belle-île et 20 personnes à Dingle ont reçu un dossier comprenant une carte format A3 de Belleîle-en-mer ou de la péninsule de Dingle (Figure 1) accompagnée d'une présentation du projet, d'un questionnaire (habitant ou visiteur, âge, sexe, condition de réalisation de la carte), d'une légende qui permet de guider la création de la carte, de feuilles vierges destinées à des notes ou des dessins et des crayons. La légende invitait à inscrire sur la carte ses activités, des sentiments, des points de vue (panorama et photographie) ainsi qu'à nommer lieux et personnes importantes. Elle invitait aussi à ajouter des commentaires divers sur les sensations, les souvenirs, les rêves que pouvaient susciter les lieux. Plusieurs types de personnes ont été interrogés : habitants, nouveaux résidents (résidents habitant les lieux depuis moins de dix ans), résidents secondaires et touristes (Tableau 1) afin d'avoir une variété de récits et d'approches. La variété des âges et des genres permet d'avoir plusieurs angles de vue et les témoignages individuels se font échos.

La plupart des visiteurs interrogés à Belle-île n’en sont pas à leur premier séjour (séjours qui varient entre un long week-end et une semaine) au contraire de la péninsule de Dingle où le

1. Quelques cartes ont été réalisées en même temps que l'entretien car les personnes n'étaient pas disponibles pour une deuxième rencontre. 


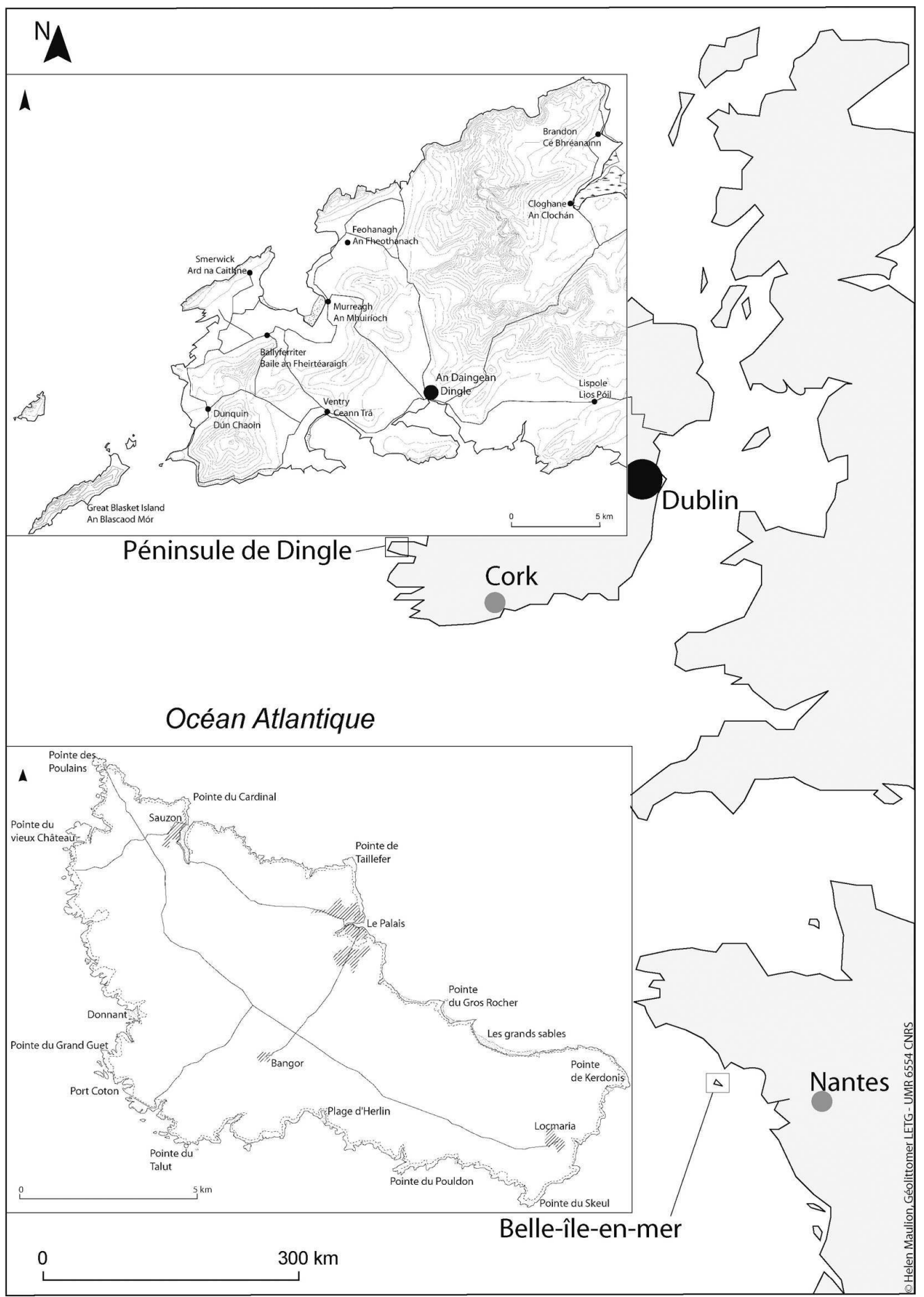

Figure 1 : Carte de localisation Map of the study areas 
tourisme est plus international et où le temps de séjour est plus court : entre une et trois nuits passées sur la péninsule.

\begin{tabular}{|c|c|c|c|}
\hline & \multirow[b]{2}{*}{ Belle-île } & \multirow[b]{2}{*}{ Dingle } \\
\hline & & & \\
\hline \multirow{5}{*}{ Tranches d'âge } & $18-30$ & $6(\mathrm{~B} 4, \mathrm{~B} 7)$ & 6 \\
\hline & $31-45$ & $4(\mathrm{~B} 10)$ & $7(\mathrm{Dl}, \mathrm{Dl} 3, \mathrm{Dl})$ \\
\hline & $46-60$ & $7(\mathrm{~B} 9, \mathrm{~B} 15)$ & $6(\mathrm{D} 19)$ \\
\hline & $61-75$ & $7(\mathrm{~B} 1, \mathrm{~B} 8, \mathrm{~B} 17)$ & $1(\mathrm{Dl})$ \\
\hline & 76 et + & $4(\mathrm{~B} 27)$ & \\
\hline \multirow{2}{*}{ Sexe } & Femme & $14(\mathrm{~B} 8, \mathrm{~B} 15, \mathrm{~B} 27)$ & $8(\mathrm{Dl}, \mathrm{Dl} 3, \mathrm{D} 19)$ \\
\hline & Homme & $14(\mathrm{~B} 1, \mathrm{~B} 4, \mathrm{~B} 7, \mathrm{~B} 9, \mathrm{~B} 10, \mathrm{~B} 17)$ & $12(\mathrm{D} 18, \mathrm{D} 11)$ \\
\hline \multirow{3}{*}{ Résident/touristes } & Résidents & $\begin{array}{l}18(\mathrm{~B} 8, \mathrm{~B} 27) \\
\text { dont } 2 \text { enfants de résidents en } \\
\text { vacances : (B9, B10) } \\
\text { et } 6 \text { nouveaux résidents : B4 }\end{array}$ & $\begin{array}{l}16(\mathrm{D} 11, \mathrm{D} 19) \\
\text { dont } 5 \text { nouveaux résidents : (D1, } \\
\text { D18) } \\
\text { et } 1 \text { temporaire }\end{array}$ \\
\hline & $\begin{array}{l}\text { Résidents secon- } \\
\text { daires }\end{array}$ & $\begin{array}{l}6 \text { (dont } 2 \text { avec des origines belli- } \\
\text { loises }) \\
(\mathrm{B} 1, \mathrm{~B} 15, \mathrm{~B} 17)\end{array}$ & \\
\hline & Touristes & 4 & $4(\mathrm{D} 13)$ \\
\hline
\end{tabular}

Tableau 1 : Caractéristiques des participants Characterising the participants

L'utilisation de la cartographie comme support d'enquête en géographie n'est pas un phénomène récent. Les avancées sur les sciences cognitives ont permis de mieux saisir les relations de l'homme à l'espace. Ainsi, la carte a souvent été utilisée par les géographes pour étudier pratiques et représentations des lieux (Barnes, 2005 ; Crouch et Matless, 1996; Péron, 1992). L'enjeu de la cartographie proposée était d'inviter à cartographier des pratiques mais aussi des expériences et des émotions. Elle est donc couplée au récit, s’inspirant de la carte de Tendre : «La carte parce qu'elle permet de reconnaître l'espace pour mieux jouir du lieu, devient alors le fétiche du Tendre » (Volvey, 2003, p. 838). Plus qu'une carte mentale où sont reportées des représentations subjectives de l'espace vécu, la carte offre une expérience créative et imaginative et un espace réflexif (Tiberghien, 2007, 2005). Les cartes paysagères deviennent des topographies imaginaires dans lesquelles le paysage, selon G. Bruno (2002) est la trace des mémoires et imaginations de celui qui a traversé le terrain et qui retient ce qui y a été assigné à chaque passage, émotions inclues.

Les cartes réalisées par les participants devenaient un objet transitionnel qui facilitait l'entretien non directif, guidant l'échange. La carte comme portion de territoire permet de se localiser. La connaissance et les souvenirs des lieux pointés du doigt surgissent simultanément. Certaines personnes ont écrit des textes pour accompagner leurs cartes où ils explicitent les symboles utilisés, situent les lieux évoqués et racontent des souvenirs. Cette écriture de soi articule émotions et souvenirs en lien avec les lieux étudiés et explicitent la relation paysagère au monde. L'entretien devenait un moment d'intimité empathique qui caractérise l'approche phénoménologique (Mucchielli et Paillé, 2005). J'étais proche du participant tout en entretenant une distance observatrice et analytique développant ainsi une « oscillation entre identification et observation » (Bondi, 2003) qui me permettait de m’engager dans l'entretien et de pénétrer dans le monde expérientiel de l'autre. Chaque entretien, non directif mais utilisant la légende et la carte comme trame, était différent et s'adaptait aux personnes interrogées en variant entre l'entretien compréhensif (Kaufmann, 2007) et le récit de vie (Bertaux, 2005). Ils ont duré entre 20 minutes et une heure et demie. La relation paysagère prenait plus ou moins forme à chaque fois. Les participants décrivent, évaluent 
et réfléchissent sur leurs expériences de tous les jours. Ils recréent des paysages en mots qu’ils me livrent le temps de l'échange.

\section{MÉThodes D’anALYSE}

Afin de ne pas perdre les formes narratives et sensibles présentes dans les entretiens pendant l'analyse des thèmes ressortant de cette enquête et d'identifier les sens donné aux expériences racontées par les participants, une double méthode d'analyse qualitative a été privilégiée. Elle associe une analyse thématique qui se base sur les entretiens transcrits, les notes de terrain et les textes écrits par les participants avec l'analyse des récits et de ses formes.

L'analyse thématique s'est inspirée de l'analyse théorique ancrée (ou Grounded Theory Analysis) développée dans les années 60 par B. G. Glaser et A. L. Strauss en réaction à la prédominance de la démarche hypothético-déductive utilisée en sciences sociales. Cette approche a évolué du point de vue des méthodes employées au fil des travaux de ces deux chercheurs mais l'idée initiale reste une source d'inspiration pour l'analyse qualitative des données. En adoptant une démarche inductive, le chercheur ne postule pas une problématique à confirmer ou infirmer ; «l'objet de recherche est défini davantage comme un «territoire à explorer » ou un phénomène à comprendre progressivement que comme question de recherche »(Guillemette, 2006, p. 37). L'objet de recherche reste ainsi provisoire dans la mesure où il peut être sujet à des évolutions au cours de l'analyse : nouvelles thématiques ou encore émergence de nouveaux paramètres à interpréter. L'analyse se fait en plusieurs étapes avec le codage des entretiens puis leur organisation en thématiques principales pour ensuite développer une théorisation (préférée à la construction d'une théorie, objectif de l'analyse théorique ancrée). L'usage du logiciel NVIVO 8.0 permet de regrouper les entretiens, leurs transcriptions et facilite le codage. Il s'agit d'entrecroiser les données et de valider les thématiques retenues par l'analyse des autres entretiens.

L'enjeu de l'analyse du récit est de restituer la voix des participants dans l'étude en axant l'analyse sur la poétique des récits et sur les images, intonations de voix et rythmiques, répétitions qui traduisent des sentiments et des émotions. L'analyse du récit ou narrative analysis considère description, évaluation et réflexion (Wyles et al., 2005). Les récits structurent l'expérience perceptive et organisent la mémoire (Riessman, 1993). Tout en replaçant l'histoire racontée dans son contexte historique, culturel et social, l'analyse du chercheur ne cherche pas l'objectivité. L'entretien peut aussi être étudié comme une performance pendant laquelle les gestes, les positions et les mots sont mis en scène pour l'auditeur. L'analyse des récits peut utiliser trois entrées différentes. L'approche littéraire étudie le caractère esthétique et métaphorique des récits; l'analyse socio-économique permet d'explorer les relations sociales, économiques et politiques présentes dans le récit; enfin, considérer la temporalité du récit permet de comprendre comment les narrateurs se situent, eux et leurs activités dans le sujet d'étude (Daiute et Lightfoot, 2004).

En développant une analyse du récit en parallèle à l'analyse thématique, la richesse poly-vocale et sensible de l'entretien est conservée et l'analyse s'en trouve enrichie.

\section{Sentir et se saisir du monde : le corps sensible}

\section{LA TACTILITÉ DU VISUEL ET LA PRÉSENCE DU/AU MONDE}

D’un paysage créé par la relation visuelle et compris comme une représentation symbolique, le paradigme moderne du paysage évolue vers le paysage comme relation sensible en mouvement. Le paysage est une des médiations entre l'homme et son milieu, celle qu'A. Berque (2000b) qualifie de sensible. S'inspirant de Le Pli de Deleuze, J. Wylie (2006) comprend le paysage comme une topographie en mouvement, composé de plis dans lesquels l'homme peut se cristalliser. C'est reconnaître au paysage son existence dans les matérialités et les sensibilités de la relation au monde. 
Le corps est un thème d'étude relativement récent en géographie malgré les travaux précurseurs des géographes humanistes (voir par exemple, Tuan, 1974; Rodaway, 1994) et plus récemment par la géographie sociale, souvent féministe qui en fait une des échelles d'étude centrale à une géographie du quotidien, de la santé ou des loisirs et qui replace le corps, ses mouvements et ses affections dans un rapport à l'espace (Besse, 2006; Fournand, 2007; Nash, 1996, 2000 ; Simonsen, 2005; Volvey, 2000). Le corps est le lieu des sens et des émotions (Davidson et Milligan, 2004), la spatialité corporelle permet de pénétrer celle du monde environnant dans une mise en contact. La dynamique de ce contact permet d'appréhender la notion de paysage non comme statique et distanciée mais comme une expérience qui développe connaissance et représentations.

Dans les entretiens, le corps n'intervient pas comme un sujet central. Les participants ont favorisé le plus souvent une approche réflexive et descriptive et ne développaient que rarement le rapport de leur corps à l'espace littoral. L'intimité du corps bien que partiellement dévoilée sur la plage, ne refaisait que peu surface pendant les entretiens. Sans être un tabou, le corps était le plus souvent oublié dans leurs récits de paysage. Et pourtant, il est présent. Les participants évoquaient des positions corporelles à travers le récit de marches, de bains de mer; ils évoquaient des sentiments, des émotions. Leurs corps étaient en mouvement sans qu'ils s'y attachent. Parfois cependant, certains se mettaient à nu. Je suggère dans les paragraphes qui suivent que le corps participe à construire la relation paysagère à travers la tactilité du visuel et à travers un processus d'incorporation du paysage. Le plus souvent solitaire, la prise de contact, dynamique, avec le monde environnant, résulte souvent en un sentiment de bien-être.

Le visuel a été largement prépondérant dans l’invention du concept de paysage, lié à sa caractérisation dans le domaine de l'esthétique et de la peinture (Cosgrove, 1984). La vue est aussi le sens le plus sollicité dans les cultures occidentales et celui qui participe le plus à la structuration de l'espace et la définition des lieux (Rodaway, 1994). Encore aujourd'hui, malgré les nombreux appels pour un concept de paysage multi sensoriel (Grout, 2003; Luginbühl, 2007), dans le rapport quotidien au paysage (Bigando, 2006), la vue joue toujours un rôle essentiel dans la définition des relations paysagères. Je voudrais réfléchir en m’appuyant sur l'étude du visible par MerleauPonty sur la dimension tactile du visuel.

L'expression « voir la mer », qui revient régulièrement dans les entretiens, comporte une dimension spatiale distanciée. Voir crée une présence, celle des terres qu'on perçoit à l'horizon, celle de la mer, celle des arbres. Voir la mer, c'est voir une matière en mouvement qui peut inspirer des sentiments variés, celui de tranquillité, de calme, mais aussi celui de plénitude, d'être bien, ou au contraire, un sentiment de peur et de fragilité face à la mer tempêtée. Au contraire, dans l'expression « regarder la mer », une dimension tactile et dans la continuité du corps entre en jeu. Le regard contient une intention, pas toujours consciente, elle établit un contact visuel avec la matière. «Le regard enveloppe, palpe, épouse les choses visibles » (Merleau-Ponty, 1964, p. 173). Le regard pénètre la matière, étudie ses mouvements, sa texture. En établissant un contact visuel par le regard, la distance à l'objet se réduit, la distance au monde environnant se réduit.

Dans l'entretien précédent, cette enseignante d'une cinquantaine d'année, connaît Belle-île depuis une trentaine d'années et y a une maison secondaire. Elle me décrit un de ses rituels, qu'elle aime renouveler l'été, sur les longues plages plates de Vazen ou de Kérel. Elle raconte la marée qui monte et la manière dont elle la regarde monter, en marchant avec elle ou en s'asseyant sur un banc de sable qui forme une île une fois entourée d'eau.

«B15 - Alors ce que j'aime bien aussi [petit sourire], bon c'est une attraction pour moi ça, c'est de regarder la mer monter. Quand je suis sur une plage! Je vais souvent d'ailleurs à Kérel pour ça ou à Vazen. [...]. Je vais sur la plage à marée basse et là je reste à peu près une heure quand la mer commence à monter et je regarde comment elle monte, la mer, et ça, c'est..., c'est très rigolo à regarder. Parce qu'elle monte par à-coups et, et ça monte très, très vite. Et on pourrait presque dire que la mer monte; on marche et la mer monte. En même temps, mais on marche tout doucement mais on la voit monter en même temps que... ça marche. C'est très curieux. Et ça j'aime bien faire ça. 
Helen - Aller voir la mer monter?

- Ah j'aime bien voir la mer monter sur la plage. [...] C'est très rigolo. J'aime bien faire ça. Alors je suis au bord de l'eau et puis j'attends qu'elle monte et je monte avec elle. Et en une heure je m'aperçois que, bah que ça va très vite. Et quelque fois même, je me mets aussi à Kérel, quand la mer est très basse, il y a des endroits qui sont complètement encastrés dans les..., dans les falaises, et on est entouré pratiquement d'eau. Et là je m'assois sur un banc de sable... et j'attends que le banc de sable soit recouvert de mer pour partir. Et là je vois effectivement ce phénomène de marée montante... [...] Et à Vazen, je fais la même chose. À Vazen il y a un endroit qui est un peu plus haut. Là quand la mer monte, la mer envahit la plage mais pas mon petit banc de sable parce qu'il est un petit peu... Alors je suis dessus et je suis bien parce qu'il y a de l'eau partout autour et moi je suis là au milieu et je ne suis pas dans l'eau. » (Entretien B15)

C'est à la fois une expérience de contemplation et une expérience tactile. Pendant la première, le mouvement de la marée rythme les pas, pendant la seconde, le corps se laisse entourer par l'élément liquide. L'interaction avec le monde est dynamique. Le littoral est un milieu en mouvement et la narratrice dans ce passage épouse ce mouvement en s'accordant avec lui. Les nombreuses répétitions, «voir la mer monter », "la mer monte », interviennent comme une métaphore qui décrit le mouvement oscillatoire des vagues et la marée montante et mouvante. L'expérience physique mène à la connaissance du milieu, elle compte et étudie le rythme des vagues.

Dans l'interaction avec le milieu littoral, le contact établi n'est pas seulement visuel et d'ordre esthétique. Merleau-Ponty (1964) écrit : «Ce n'est pas l'œil qui voit mais ce n'est pas l'âme, c'est le corps comme totalité ouverte. » Le toucher est un sens que l'on oublie alors qu'il est actif en permanence, pénétrant la matière du monde.

\section{LE CORPS SENSIBLE EN MOUVEMENT : MATÉRIALISATION ET INCORPORATION}

La relation paysagère est sensible, elle est construite à travers la performance de soi dans le monde : une mise en scène où le corps est percevant et sensible, allant d'affects en affects, dans un mouvement à la fois kinesthésique et ontologique. Les autres sens animent aussi la relation aux paysages. Les odeurs sont associées le plus souvent à une temporalité : l'odeur des ajoncs qui évoque celle de la noix de coco, est assimilée au printemps. L'odorat rappelle aussi aux personnes interrogées la présence d'une vie non humaine, végétale (odeur de l'ombre) et animale (traces des moutons) et permet d'établir un contact avec le reste du monde environnant en ré-établissant ces présences autres.

Le paysage devient une topographie sensuelle dont le corps épouse et enregistre, parfois dans l'effort, les formes. Le contact établi avec la verticalité macro scalaire de la falaise ou de la montagne et les anfractuosités micro scalaires oblige le corps à trouver sa place dans la morphologie rocheuse. Le corps qui descend la falaise doit trouver les creux ... «Quand on était jeune..., la première fois que j'y suis allé [à la pêche], [mon père] m'a fait descendre là. [...] Mais c'est pas facile du tout. Vraiment pas de place! On a le pied, comme ça, pour mettre le pied à un moment [montre avec sa main qui imite une pointe de pied] » (Entretien B9).

...Et appuyer le dos à la solidité de la roche quand la mer arrive en paquet et que la vague vient fouetter le corps debout et exposé comme le raconte cet enseignant qui a grandi en partie sur l'île. Le corps est une topographie en mouvement, enregistrant la morphologie des roches, le mouvement de vagues. Merleau-Ponty (1964) développe une ontologie de la chair qui entrelace le corps au monde et le monde au corps. Le corps devient paysage et le paysage devient incarné. Le philosophe note que "l'épaisseur du corps, loin de rivaliser avec celle du monde, est au contraire le seul moyen que j'ai d'aller au cœur des choses, en me faisant monde et en les faisant chair » (1964, p. 176). Cette ontologie de la chair lui permet de dépasser le conflit entre une perception subjective et l'objet perçu. Le contact corporel, sensoriel et sensuel créé par l'individu avec l'environnement résulte en une spatialité entrelacée et communicante. Cela conduit à explorer une 
géographie incarnée et donc en mouvement qui interroge le corps comme performant la relation paysagère. Les termes d'énergie' et de 'force' reviennent dans les entretiens. Ils qualifient l'impression de force et de mouvement que les participants ont au bord des falaises. Le terme impression n'est pas anodin ici. Il peut évoquer une impression corporelle, à la manière d'un tatouage. C'est ainsi qu'à l'approche de la mer en tempête, le corps au bord de la falaise se plie sous la force des éléments, le vent modèle le corps comme il modèle les vagues, et les embruns viennent mouiller le corps replié.

Le contact avec l'environnement est corporel dans l'entretien qui suit aussi. Souvent, quand les participants évoquent une pratique renouvelée, ils utilisent la seconde personne du singulier comme s'ils se détachaient de leur expérience pour pouvoir la raconter et aussi la faire partager. Le «tu », c'est eux, et c'est moi aussi, qui écoute et qui peut m’identifier à leur expérience. Par le retour à la première personne du singulier, ce jeune homme d'une vingtaine d'année, qui découvre l'île en bateau et qui vient de trouver un travail sur l'île dans le bâtiment, évoque un souvenir et un sentiment précis.

«B7 — Là, le rocher que j’ai coché, je sais pas... C'est un peu compliqué de monter dessus, mais là-bas t'as vraiment l'impression d'être... si tu veux vraiment rester dessus pendant un petit bout de temps, tu restes toute la marée.

Helen - Humm.

- Tu attends à peu près six, sept heures pour que tu puisses repartir du rocher.

— Tu t'es déjà...?

- Bah une fois, j'étais resté bloqué, mais j'ai trouvé ça très bien car comme c'est un gros rocher, il y a plein d'enclaves... J'étais bien là-dessus. » (Entretien B7)

Ainsi, le sentiment d'être bien revient régulièrement dans les entretiens. Il est recherché et les participants ont souvent un lieu qui leur permet de retrouver une forme de sérénité ou de paix. Ce sentiment de bien-être est le plus souvent développé dans la solitude. Ainsi, cette jeune femme, qui travaille à Dingle depuis quelques années, évoque à plusieurs reprise la plage de Ventry : "it's a place where I go back, not feeling you know in top form, I'd go back and walk on from Ventry Beach, and I always feel better» (entretien D1). Chacun a son endroit propre à lui. Cela évoque la définition qu'en donne M. de la Soudière (2008, p.13) : «Un lieu pour moi, c'est là où on se pose et se repose, se refait ». Cependant, le lieu n'appelle pas seulement à la pause mais aussi au mouvement. Il faut parler de passage, créé ici par la marche, l'escalade d'un rocher comme dans l'entretien précédant ou par la nage comme dans l'extrait d'entretien qui suit. Cet homme, d'une quarantaine d'années, est d'origine anglaise et habite sur la péninsule depuis huit ans. Il raconte que chacun des lieux où il nage correspond à différentes manières de nager et à des périodes différentes de l'année. Il a cartographié toutes les plages en détaillant leurs particularités, quand il y nage et certains souvenirs précis (Figure 2). Il raconte une de ces nages dans le paragraphe suivant :

"Sometimes when I swim [in Dún Séanna], I swim all the way from here, right out to the end and I leave around the corner so I arrive right down under the cliff of the old iron age fort that is here. Nobody knows I'm there, um... you know it's quite dangerous in some ways. In the other hand, there's an amazing sense of solitude and bit of pace, being in nature and surrounded by just water and rocks and birds and you could sit there. » (Entretien D18)

Dans ce récit comme dans le premier et d'autres, on retrouve le même schéma expérimental, voire initiatique ou picaresque. Il y a une phase de progression vers l'endroit requis : à la nage ou à pied, qui demande un effort physique, une pénétration de l'épaisseur qui nous distance du lieu. Le corps en mouvement se fraie un passage vers le lieu qui permet à l'individu d'être et mieux encore d'être bien. Le corps se déploie dans l'environnement dans un mouvement 


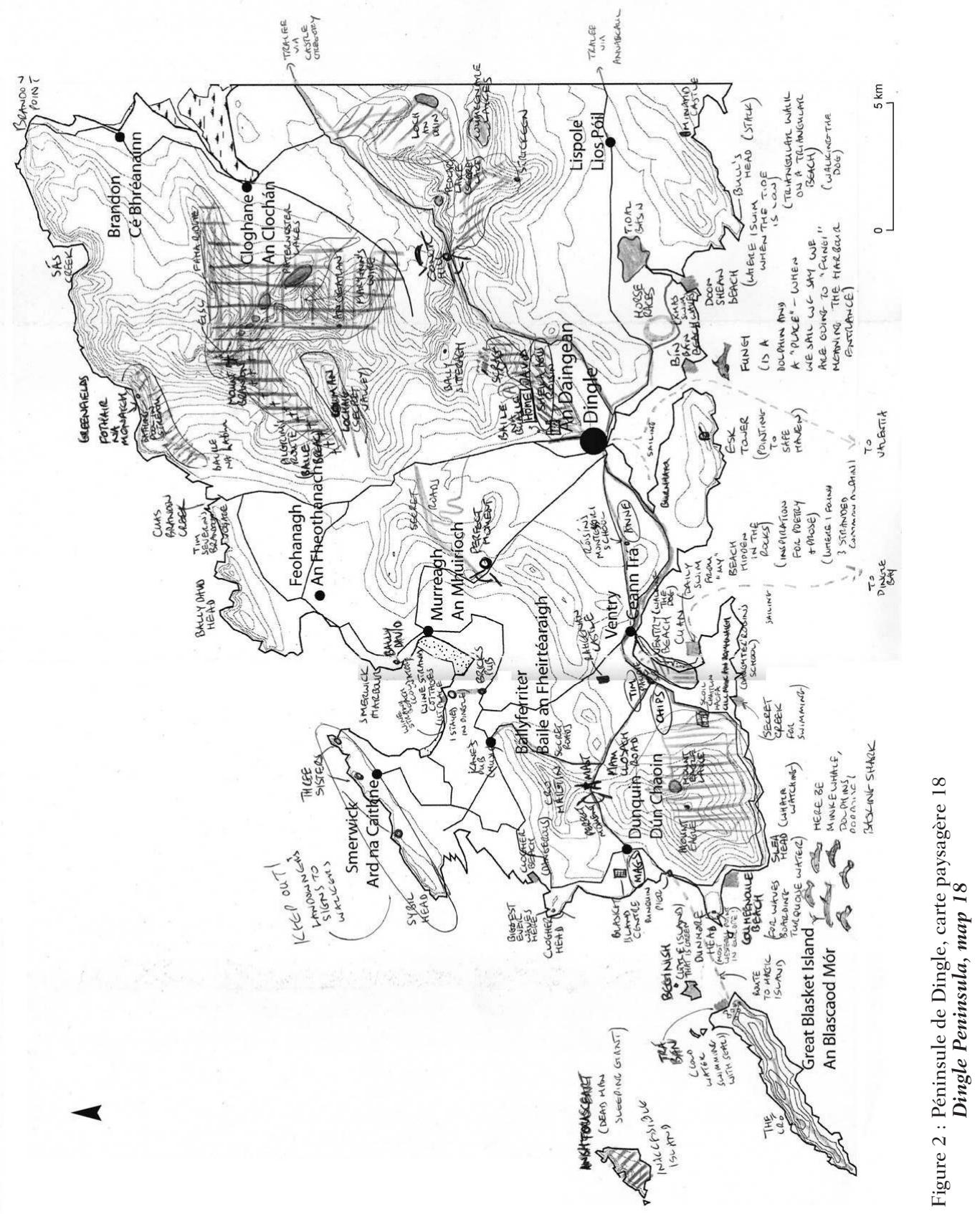


inverse à celui d'incorporation paysagère. Il s'isole vers une forme d'extrémité littorale : falaise et rochers. La traversée aérienne ou liquide mène curieusement vers la matière rocheuse, solide, socle rassurant. Le corps s'immisce dans ses creux : «to find a place just for me to be there » dit une touriste canadienne (entretien D13) en s'asseyant à l'extrémité de la péninsule de Dingle. Le corps en se recroquevillant dans les creux de la pierre s’immobilise. Il vient au contact des éléments dans une liminalité qui semble permettre l'immersion matérielle. La relation paysagère est nourrie par le mouvement entre l'incorporation paysagère (Bigando, 2006) et la matérialisation du corps. La place de la transition initiatique dans les récits des participants me semble contenir plus que l'idée d'existence, celle de devenir et de construction. Le sentiment de bien-être, ponctuel et associé à l'expérience vécue, semble dépendre de la présence simultanée du monde environnant et du corps ayant trouvé sa place dans cet environnement. Cela produit un sentiment d'harmonie et de paix favorisé par l'écoute du monde et sans doute le sentiment d'être présent et de participer à la dynamique élémentaire et vivante du littoral.

L'attention s'est portée dans cette partie sur le corps sensible comme animateur de la relation au monde, puis sur le mouvement d'assimilation entre les formes matérielles et le corps. Ces relations paysagères sont sensibles, sensuelles et émotionnelles et entraînent un sentiment de bien-être. Le contact avec le monde environnant est créé dans l'instant, entrelacé au corps en mouvement. Il caractérise une relation qui n'est pas passive et contemplative mais engagée et réflexive sur le milieu habité.

\section{Engagement et attachement : habiter et paysager son monde}

Dans cette dernière partie, il s'agit de continuer à explorer les relations aux littoraux et aux terres de Belle-île-en-mer et de la péninsule de Dingle en associant le concept d'habiter et l'idée d'engagement. Ces milieux 'refuges' et pourtant fortement touristiques pendant la saison estivale (500 000 visiteurs par an à Belle-île ${ }^{2}$ ), sont appréciés dans la mesure où ils offrent un contact avec une nature qui semble préservée, encore sauvage. C'est le rôle que jouent l'océan et la montagne. Cependant, sans être seulement consommateur, le rapport individuel établi avec ces milieux littoraux mais aussi ruraux est de l'ordre de l'engagement qui peut être aussi critique et qui souvent porte une vision et se projette dans le futur ${ }^{3}$. Le concept d'habiter a une dimension ontologique et territoriale, qui s'inscrit dans la mobilité (Stock, 2004) et contribue à la relation paysagère en tant que « l'être-là » est perceptif et actif dans le monde (Hoyaux, 2000). Les relations paysagères narrées dans les récits des participants ne sont pas construites sur un mode descriptif et distancié. Les récits paysagers présentent un engagement qui est à la fois actif, réflexif et réceptif avec le monde. La relation paysagère est ainsi en construction permanente et anime un mouvement entre savoir vernaculaire (noms de lieux, de plantes, connaissance des lieux dans ses moindres recoins) et les connaissances scientifiques, entre représentations artistiques, touristiques ou politiques et une expérience créative : écrite ou physique, pendant laquelle les personnes essayent de ré-établir un contact avec la dynamique écouménale.

\section{RÉ-INVENTION DE LA RELATION AU MONDE}

La relation paysagère à l'échelle individuelle est ainsi construite socialement dans un engagement avec les lieux habités et les personnes qui les habitent. A la fois hédonistes et critiques, les participants forment des récits complexes où le paysage surgit dans leur rapport avec leur milieu. Il s'agit de suggérer l'aspect créatif du rapport à son milieu, comme une forme d'invention du

2. Source CCBI (Communauté de Communes de Belle-île).

3. Certainement, à Belle-île, ce projet passe par la sensibilisation à l'environnement, aux milieux et aux traditions de l'île. A Dingle, ce projet de développement est plus difficile à situer, il est peu évoqué par les participants sauf par certains qui essayent de faire surgir une réflexion sur ces questions face aux propositions et aussi à l'absence de propositions des acteurs politiques. 
quotidien (De Certeau, 1990) et une forme de re-création de soi (Équipe MIT, 2002) à travers les expériences de loisir ou de tourisme dans «le territoire de l'hors-quotidien » (Bourdeau, 2003) qu'est le littoral pour nombre des participants. La confrontation dynamique avec le monde, mode de connaissance et de savoir est réinventée par l'expression créative, poétique et artisane. Intervient le pouvoir de l'imagination métaphorique. Les bois flottés, populaires pour leur formes sculptées par la mer aujourd'hui, recherchés avant comme bois de chauffage, font surgir des formes chez une des participantes, une femme à la retraite, revenue vivre sur l'île après y avoir grandi : "C'est un bois, enfin, ce qu'on appelle un bois flotté [...] quand je voyais le bois, tout de suite je voyais la forme que ça avait [...] il suffisait d'enlever deux ou trois bouts de choses pour faire ressortir la forme. Pour moi, c'était comme un accouchement et on enlève le surplus et... la quintessence de la sculpture apparaît» (Entretien B8).

Ainsi, une forme de jeu créatif et re-créatif se met en place, associant l'être dans son environnement. Ces «manières de faire » le paysage permettent de nourrir le contact et de s'engager physiquement et imaginairement avec le monde.

De même, la marche accompagne la recherche d'images poétiques que les participants me livrent. Ainsi, ce poème, réflexif sur le passage et le mouvement, est écrit après des promenades et des nages sur la plage de Ventry.

"-All across the bay, where on Sunday I had stretched to swim its diameter, the sea was punctuated by small explosions of the gannets as they hit the water. I strained to see those other animals, the dolphins that might be attracted by the same concentration of fish. To no avail.

- Who walks this path today?

- Unsure, my feet follow smoothed sandstone.

- Strata on edge.

- A way through raggedy rocks separating striped strands. » (Notes, D18)

Ces images révèlent un autre aspect de leur engagement avec l'altérité du monde, à la fois joueur et créatif dans la contemplation, le mouvement mais aussi dans l'interrogation. C'est ainsi que cette femme, d'une cinquantaine d'année, qui habite Dún Chaoin depuis plus de vingt ans me raconte comment elle descend parfois dans la crique près de chez elle. Le 'cuisin' est un lieu particulier pour elle puisqu'il la connecte aux îles Blasket; c'est là que les habitants de l'île accostaient avec leur naomhóg, canots de toile goudronnée. Une rivière s'y écoule depuis le Mount Eagle, et elle me raconte: "The river, there's something about the river. I used to spend, I don't spend as much time now but I still do it but I always build bridges across. I don't know why I do that. Mhm. I build a little, the stones, just putting the stones together from this side over, because over to the other side, then you can walk up towards the heritage centre and to the main road. It's the Dingle way. And I don't know. It was a time I had a lot of time at home and I used to go digging there. I don't know why I used to... You can spend hours, just building bridges. But it wasn't so much... it was the bridges, but also putting the stones right. Mhm So that people would be able to get across. I'm just... [laughs]. I don't know... I'd just... They're talking about putting a permanent one in, the County Council, I hope they don't. Mhm. I don't want a permanent struct... It wouldn't be right because half the thing is getting across » (Entretien D19).

Comme elle le souligne, elle construit ce pont de pierre à la fois pour prolonger le sentier de randonnée mais surtout pour le geste d'associer une pierre à une autre, de la bonne manière. De cette performance, elle construit quelque chose, elle nourrit surtout sa relation à la crique, à l'île des Blaskets et à son corps.

Construire dans le paysage peut s'assimiler à une appropriation de l'espace. Il s'agit ici aussi de l'idée de bien-être entraîné par la présence dans le lieu, la recherche d'une création harmonieuse et éphémère qui bénéficiera à d'autres. Il y a une invention et un renouvellement permanent de la relation individuelle au paysage, qui s'ajoutent à la construction sociale du territoire de l'habitant. 


\section{Trajectoire de VIE ET ÉVOLUTION DE LA RELATION PAYSAgÈre}

Habiter à Belle-île ou dans la péninsule de Dingle, faire le choix d'un lieu de vie comme cela a été le cas pour beaucoup des participants (résidents secondaires ayant des origines locales, ou venant depuis plus d'une dizaine d'années, nouveaux résidents), c'est vivre sur des territoires littoraux, touristiques et ruraux, souvent attractifs car ils offrent une réponse au besoin de nature (Donadieu, 1994). Le sentiment de « vivre ici » s'accompagne souvent dans les entretiens de celui d'appartenance ou d'appropriation, tous les deux participant à celui d'attachement aux lieux. Les récits, autobiographiques, entrelacent la relation paysagère en construction à la trajectoire de vie individuelle et les cartes révèlent souvent ces trajectoires de vie à travers la ou les maisons qui y sont reportées (Figure 3). Ces lieux de vie intimes forment la porte d'entrée vers les paysages. Les participants agencent leur parcours de vie avec certains des lieux de l'île ou de la péninsule. L'évocation de la maison ou des maisons, seconde échelle de la relation paysagère après celle du corps, est une manière de construire leur territoire associée chez certains à un sentiment d'appartenance : " 24 years I'm doing now, you know, I'm in the world where I belong, in the landscape here, in the people here ( entretien D19). Un sentiment d'accomplissement accompagne ces mots, cette femme d'une cinquantaine d'années utilise le terme 'doing' qui traduit une action en cours alors qu'il serait plus commun de dire qu'elle vit (to live) à Dunquin (ouest de la péninsule) depuis 24 ans. Ce sentiment d'appartenance est loin d'être présent dans tous les récits des participants. Cependant, il traduit l'attachement à certains lieux de la péninsule ou de l'île.

Cet attachement est aussi exprimé à travers l'exploration et la découverte de lieux, de formes, d'histoires. Chaque promenade est l'occasion d'une découverte porteuse d'une petite excitation qui renouvelle et complexifie la relation aux lieux, ainsi que le raconte cet homme, photographe et résident secondaire : "Tous les ans, je découvre un chemin que je n'ai jamais fait [...] si on se penche un peu plus sur une falaise, on va découvrir quelque chose en bas qui est étonnant, parce que ce jour là, on s'est penché un peu plus bas au bord d'une falaise» (entretien B1).

Ces expériences de découverte nourrissent, enrichissent la relation paysagère et confortent l'attachement à l'île ou la péninsule. Ainsi, le sentiment d'appartenance qui est associé à l'idée de construction territoriale montre ici comment la relation paysagère, des habitants, des résidents secondaires et des touristes interrogés, naît de l'appropriation d'un lieu, de la connaissance qu'on peut en avoir, d'une exploration constante et de découverte sans fin. Cette relation paysagère qui est réflexive, diffère cependant selon les participants. Les récits des touristes sont imprégnés d'impressions brutes provenant d'une rencontre courte avec les paysages de l'île ou de la péninsule. Leur relation aux paysages repose sur leur découverte immédiate du milieu. Celles des habitants sont souvent au contraire passées à travers les mailles de l'histoire et de leur histoire personnelle. Les lieux sont empreints de souvenirs et ils participent à la relation au monde, à la manière de le vivre. Les récits des nouveaux résidents (Dingle et Belle-île) et des résidents secondaires (Belleîle) révèlent une autre dimension : celle du choix de vie. Plusieurs situations existent : retour sur l'île au moment de la retraite, installation définitive des résidents secondaires, arrivée de nouveaux résidents. Ainsi, la trame temporelle change dès que les récits paysagers évoquent les itinéraires de vie des participants, l'évolution de leur relation avec le milieu ou avec les formes paysagères. Les expériences et les souvenirs sont replacés dans une trajectoire de vie. Cette femme, retraitée, qui était femme d'agriculteur à Belle-île et l'aînée d'une famille nombreuse me dit avoir commencé à apprécier les paysages littoraux de l'île avec la retraite, quand elle a eu le temps de se promener le long des sentiers côtiers (Entretien B27). La côte si proche n'était que rarement objet de promenade avant; elle n'en avait pas le temps.

Ces découvertes et le déploiement d'un contact inventif et attentif au monde environnant transforment la pratique quotidienne des lieux et en favorisent l'appropriation tout en permettant la construction d'une relation au monde réflexive et inventive mais aussi critique.

Cette relation n'est plus seulement paysagère, mais territoriale et influence la perception sensible des lieux et l'évolution de la relation paysagère. 
Helen Maulion
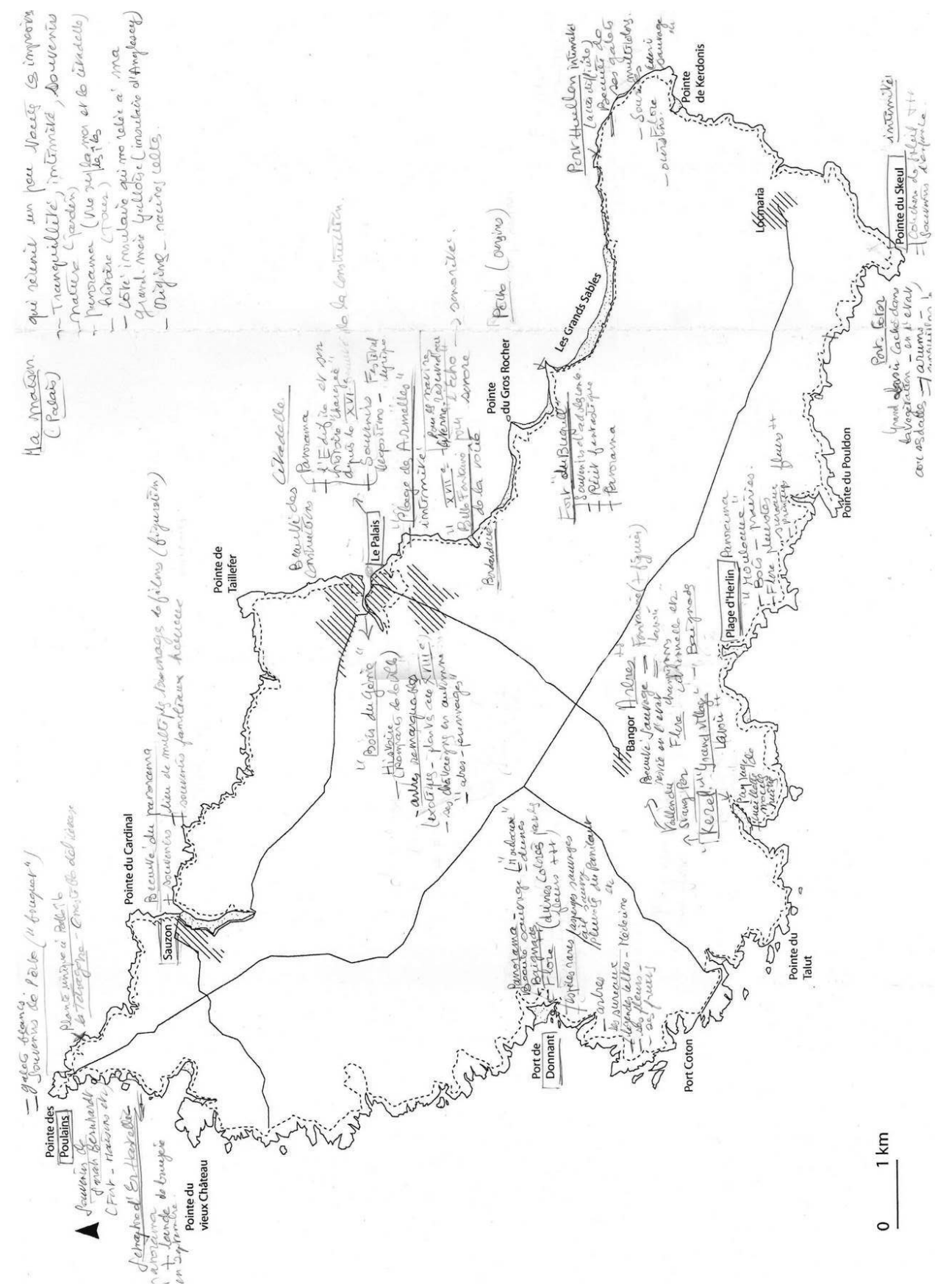

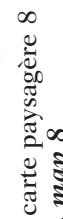


La manière dont cet homme, qui est né à Dingle et y a vécu toute sa vie (il a maintenant plus de 70 ans), explorait la péninsule a évolué au fil du temps. Des explorations avec ses jeux d'enfant et d'adolescent, il a ensuite chassé, travaillé comme postier, et depuis une vingtaine d'années, il s’intéresse à la géologie, à la faune et à la flore de la péninsule, ce qui lui a permis de renouveler son approche de la péninsule. Il me raconte aussi que le sentiment de vivre dans un endroit magnifique et agréable s'accompagne aujourd'hui de l'irritation face aux aménagements du territoire qu'il critique : multiplication des constructions, destruction de falaises, déficit d'approche globale et durable des plans de développement. Il souligne la manière dont sa connexion à certains lieux de la péninsule a disparu avec des aménagements :

«A particular site was the Pedlar's Lake always sure to revive me if down. [...] A local politician organised converting the Pedlar's Lake into "put in take out" fishery, a crass form of angling not even wanted by the local anglers who were worried [...] This site was pristine and it was a mountain group who raised the alarm seeing a rough path made and posts and advertising signs put up. While there was no gross damage to the morphology of this geologically significant site, the feeling this created was not unlike a room that has been burgled. Indeed since this was ended I have not felt quite the same about the lake. " (Notes D11)

Cette critique de l'évolution des milieux et des politiques publiques de développement se retrouve à Belle-île sur plusieurs thématiques. Les constructions de nouvelles maisons, détachées, entourées de larges jardins brisent l'harmonie des hameaux. Le thème de l'enfrichement, due à la transformation des pratiques (la lande ne sert plus de litière pour les animaux) et à la déprise agricole, revient à plusieurs reprises dans les entretiens. Ce jeune homme, qui travaille dans l'environnement, relève que la généralisation de l'enfrichement peut finir par couper la vue sur l'océan : " même sur le plateau, on a beaucoup de friches à prunelliers qui se développent, et dans certains coins [...], on a des voiles de végétation en fait qui nous empêche de voir la ligne d'horizon et d'apprécier un paysage » (entretien B4), alors que l'île était caractérisée par son plateau dénudé. Comme ce résident secondaire et agronome récemment à la retraite, il suggère d'encourager l'activité agricole sur l'île. Ces idées, nées à la fois de la pratique et de l'observation du milieu participent de la réflexion sur l'habiter et la gestion du milieu qui devrait tendre vers un bien-être non seulement individuel mais social (Luginbühl, 2006), appréhendable à travers une approche territorialisée de la relation paysagère.

\section{Conclusion}

Performances et récits nourrissent la relation paysagère, qui est sensible et esthétique mais qui est aussi métaphorique. Les relations paysagères ne sont pas définies seulement par rapport à des représentations. S'intéresser aux connexions sensibles avec le littoral nécessite une variation d'échelles à la fois spatiale et temporelle. La première considérée dans cet article est celle du corps qui permet une mise en contact sensible avec le milieu littoral, puis vient celle du territoire. La relation paysagère se nourrit de ces multiples échelles spatiales. Elle est animée aussi par le rapport au temps à la fois celui de l'instant perceptif et celui de la mémoire de differentes expériences constructrices de soi. Les récits proposent des itinéraires de vie qui permettent de comprendre la formation et l'évolution de leur relation paysagère, intime et sensible, mais aussi sensuelle, puisqu'elle invite à renouveler les plaisirs du contact avec le monde environnant. Les formes et le mouvement littoral offrent support et métaphore pour la relation paysagère qui est dynamique et liminale. Celle-ci s'apparente à un mouvement pénétrant, reposant sur l'expérience sensible du milieu de vie, basée sur la recherche d'harmonie et de liberté. Elle est en devenir et modelée par une forme d'engagement qui inscrit l'individu au sein de l'écoumène qu'il participe à animer. La relation paysagère individuelle est aussi sociale car elle tend vers un intérêt commun : celui du bien-être accessible à travers l'engagement physique, affectif et créatif avec le milieu. 
Finalement, penser le paysage en terme de relation phénoménologique permet d'approcher ce que Augustin Berque (2008) appelle « la pensée paysagère» à travers une géographie ontologique et sensible en mouvement.

\section{bibliographie}

Barnes (A.), 2005. - «Geo/graphic mapping », Cultural geographies, vol. 14, nº 1, p. 139-147.

Berque (A.), 2008. - La pensée paysagère, Paris, Archibooks-Sautereau Editeur, 111 p.

—, 2000a. - Ecoumène. Introduction à l'étude des milieux humains, Paris, Belin, 271 p.

—, [1990] 2000b. - Médiance. De milieux en paysage, Paris, Belin, 159 p.

—, 1996. - Etre humain sur la terre, Paris, Gallimard, 212 p.

Bertaux (D.), 2005. - Le récit de vie, Paris, Armand-Colin, 127 p.

Besse (J.-M.), 2006. - « L'élan du paysage. Premières notes sur la danse et l'écriture », Les carnets du paysage - Comme une danse, vol. 13-14, p. 11-19.

Bigando (E.), 2006. - La sensibilité au paysage ordinaire des habitants de la grande périphérie bordelaise (communes du Médoc et de la basse vallée de l'Isle), thèse de doctorat de géographie sous la direction de G. Di Méo, Bordeaux, Université Montaigne, 490 p. [http://tel.archives-ouvertes.fr/docs/00/14/84/40/PDF/ TheseEVA_web.pdf]

Bondi (L.), 2003. - «Empathy and Identification : Conceptual Resources for Feminist Fieldwork », ACME : An International E-Journal for Critical Geographies, vol. 2, n 1 [http://www.acme-journal.org/vol2/Bondi. $p d f]$.

Bourdeau (P.), 2003. - Territoires du hors-quotidien : une géographie culturelle du rapport à l'ailleurs dans les sociétés urbaines contemporaines; le cas du tourisme sportif de montagne et de nature, HDR, Grenoble, Université Joseph Fourier, 269 p.

Bousquet (B.), 1990. - « Du littoral. Essai d'identification », Cahiers Nantais, vol. 35-36, p. 77-98.

Bruno (G.), 2002. - Atlas of Emotion, London, Verso, 524 p.

Buttimer (A.), 1976. - « Grasping the dynamism of lifeworld », Annals of the Association of American Geographers, vol. 66, n² 2, p. 277-292.

Corbin (A.), 2005. - Le ciel et la mer, Paris, Bayard, 121 p.

—, 1988. Le territoire du vide. L'Occident et le désir de rivage, 1750-1840, Paris, Flammarion, 407 p.

Cosgrove (D. E.), [1984] 1998. - Social formation and symbolic landscape, Madison, The University of Wisconsin Press, 293 p.

Crouch (D.), Matless (D.), 1996. - Refiguring Geography : Parish Maps of Common Ground. Transactions of the Institute of British Geographers, vol. 21, n 1, p. 236-255.

Daiute (C.), Lightfoot (C.), 2004. - Narrative Analysis : Studying the Development of Individuals in Society, London, Sage, $320 \mathrm{p}$.

Davidson (J.), Milligan (C.), 2004 - « Editorial : Embodying Emotion Sensing Space : Introducing emotional geographies », Social and Cultural Geography, vol. 5, n 4, p. 523-532.

Dardel (E.), [1952] 1990. - L'homme et la terre, Paris, CTHS, 201 p.

De Certeau (M.), [1980] 1990. - L'invention du quotidien. 1. arts de faire, Paris, Folio, 350 p.

De la Soudière (M.), 2008. - Lignes secondaires, Grânes, Creaphis Editions, 126 p.

Donadieu (P.), 1994. - « Pour une conservation invention inventive des paysages », dans Berque (A.) (dir.). Cinq propositions pour une théorie du paysage, Seyssel, Champ-Vallon, p. 51-80.

Équipe MIT, 2002. - Tourismes 1. Lieux communs, Paris, Belin, 320 p.

Fournand (A.), 2007. - « Le corps, objet central des Feminist Geographies », L'espace géographique, vol. 36, $n^{\circ} 4$, p. 376-377.

Grout (C.), 1999. - Écouter le paysage, Strasbourg, École nationale des arts décoratifs, 69 p.

Guillemette (F.), 2006. - «L'approche de la Grounded Theory; pour innover? » Recherches qualitatives, vol. 26, $\mathrm{n}^{\circ} 1$, p. 32-50. 
Hoyaux (A.-F.), 2000. - Habiter la ville et la montagne: Essai de géographie phénoménologique sur les relations des habitants au lieu, à l'espace et au territoire Exemple de Grenoble et de Chambéry), Thèse, Grenoble, Université Joseph-Fourier, 720 p.

Kaufmann (J.-C.), 2007. - L'entretien compréhensif (2e édition), Paris, Armand-Colin, 126 p.

Luginbühl (Y.), 2007. - « Pour un paysage du paysage », Économie Rurale, vol. 297-298, n 1, p. 23-40.

—, 2006. - « Paysage et bien-être individuel et social » dans Conseil DE L’Europe - Paysage et développement durable : les enjeux de la Convention Européenne du paysage, Strasbourg, Éditions du Conseil de l'Europe, p. 31-55.

—, 2001. - « La demande sociale de paysage », Conseil National de Paysage, Rapport de la séance inaugurale, Ministère de l'Aménagement du Territoire et de l'Environnement, p. 7-29.

Merleau-Ponty (M.), [1964]. - Le visible et l'invisible, Paris, Tel, 361 p.

Mucchielli (A.), Paillé (P.), 2005. - L'analyse qualitative en sciences humaines et sociales, Paris, ArmandColin, $211 \mathrm{p}$.

NASH (C.), 2000. - « Performativity in practice : some recent work in cultural geography », Progress in Human Geography, vol. 24, n 4, p. 653-664.

—, 1996. - « Reclaiming Vision : looking at landscape and the body », Gender, Place and Culture, vol. 3, $\mathrm{n}^{\circ} 2$, p. 149-170.

Péron (F.), 1992. - «L'̂̂le, espace culturel », Géographie et cultures, 1(2), vol. 2, p. 3-33.

Riessman (C.), 1993. - « Narrative Analysis », Qualitative Research Methods, vol. 30, Londres, CA : Sage.

Rodaway (P.), 1994. - Sensuous geographies. Body, Sense and Place, Londres, Routledge, 198 p.

Simonsen (K.), 2005. - « Bodies, sensations, space and time: the contribution from Henri Lefèbvre », Geografiska Annaler, vol. 87B, n 1, p. 1-14.

Sтоск (M.), 2004. - «L'habiter comme pratique des lieux géographiques », EspacesTemps.net, Textuel, [http:// espacestemps.net/document 1138.html].

Tiberghien (G.-A.), 2007. - Finis Terrae. Imaginaires et imaginations cartographiques, Bayard, Paris, 204 p. —, 2005. - La nature dans l'art, Arles Actes Sud, n.p.

Thrift (N.), 2004. - «Intensities of feeling : towards a spatial politics of affect », Geografiska Annaler, vol. $86 \mathrm{~B}, \mathrm{n}^{\circ} 1, \mathrm{p} \cdot$. 57-78.

TuAn (Y. F.), 1974. - Topophilia : a study of environmental perception, attitudes, and values, Londres, Prentice-Hall, $260 \mathrm{p}$.

Volvey (A.), 2002. - «Sexualité », dans Lévy (J.), Lussault (M.) (dir.), Dictionnaire de la géographie et de l'espace des sociétés, Paris, Belin, p. 837-838.

—, 2000. - « L'espace, vu du corps », dans Lévy (J.), Lussault (M.) (dir.), Logiques de l'espace, esprit des lieux. Géographies à Cerisy, Paris, Belin, p. 319-332.

White (K.), 1994. - Le Plateau de l'Albatros, Paris, Grasset, 367 p.

Wyles (J. L.), Rosemberg (M. V.), Kearns (R. A.), 2005. - « Narrative analysis as a strategy for understanding interview talk in geography research », Area, vol. 37, n 1, p. 89-99.

WyLie J., 2007. - Landscape, Londres, Routledge, 246 p.

—, 2006. - « Depths and folds : on landscape and the gazing subject », Environment and Planning D : Society and Space, vol. 24, n 4, p. 519-535.

Cet article a été reçu le $1^{e r}$ mars 2009 et définitivement accepté le 20 juin 2009. 
